\title{
Acciones de participación social, identidad y emociones de estudiantes chilenos de una universidad privada
}

\author{
Loreto Villagrán Valenzuela \\ Universidad de Concepción, Concepción, Chile \\ Email: lorevillagran@udec.cl \\ Carlos Reyes Valenzuela \\ Universidad Andina Simón Bolívar, Quito, Ecuador \\ Email: carlos.reyes@uasb.edu.ec \\ Annia Wlodarczyk \\ Universidad Católica del Norte, Antofagasta, Chile \\ Email: anna.wlodarczyk@ucn.cl
}

\begin{abstract}
Resumen: Chile presenta una larga data de movilizaciones relacionadas con el acceso a la educación, adquiriendo en la última década gran notoriedad el Movimiento Estudiantil (ME) que ha vinculado a estudiantes del sistema público o de universidades pertenecientes el Consejo de Rectores en quienes se han focalizado principalmente las investigaciones. El objetivo principal de este estudio fue analizar la relación entre la identidad colectiva (IC), identidad politizada (IP), emociones (E) y acciones de participación social (PS) en estudiantes de una universidad privada. En el estudio participaron 224 estudiantes de la carrera Psicología de una universidad privada chilena, con edades entre 19 y 33 años. Los resultados identifican mayores acciones de PS en hombres, con alta influencia de las emociones positivas y negativas en activar tales acciones. Por otro lado, en estudiantes que perciben una IC, las emociones de esperanza, vergüenza e impotencia median las acciones de PS y en los estudiantes con una alta IP, solo la emoción de la vergüenza media la participación. Se discuten las implicaciones de estos hallazgos y de las posibilidades y limitaciones que se presentan para los estudiantes universitarios participar en el ME desde una universidad privada.
\end{abstract} ciología

Palabras claves: Movimiento estudiantil; participación; emociones; identidad colectiva; identidad politizada; so-

\section{Actions of social participation, identity and emotions of Chilean students of a private university}

\begin{abstract}
Chile has a long history of mobilizations related to access to education, and in the last decade the Movimiento Estudiantil (ME) has acquired great notoriety, linking students from the public system or from universities belonging to the Consejo de Rectores (Council of Rectors) on whom research has focused mainly. The main objective of this study was to analyze the relationship between collective identity (IC), politicized identity (IP), emotions (E) and social participation actions (PS) in students of a private university. The study included 224 students from the Psychology degree program at a private Chilean university, aged between 19 and 33. The results identify greater PS actions in men, with high influence of positive and negative emotions in activating such actions. On the other hand, in students who perceive an IC, the emotions of hope, shame and helplessness mediate the actions of PS and in students with a high IP, only the emotion of shame mediates participation. The implications of these findings and the possibilities and limitations presented for university students to participate in the ME from a private university are discussed.
\end{abstract}

Keywords: Student movement; participation; emotions; collective identity; politicized identity; sociology 


\section{Ações de participação social, identidade e emoções de estudantes chilenos de uma universidade privada}

Resumo:Chile tem uma longa história de mobilizações para exigir o acesso à educação, adquirindo na última década grande notoriedade o Movimento Estudantil (ME), que há ligado a alunos da rede pública ou de universidades do Conselho de Reitores em quem têm-se focado principalmente as pesquisas. O principal objetivo deste estudo foi analisar a relação entre a identidade corporativa (IC), identidade politizada (IP), emoções (E) e ações de participação social (PS) em estudantes de uma universidade privada. No estudo participaram 224 estudantes do curso de Psicologia de uma universidade privada chilena, com idade entre 19 e 33 anos. Os resultados identificam maiores ações de PS em homens, com alta influência de emoções positivas e negativas na ativação de tais ações. Por outro lado, em estudantes que recebem um IC, as emoções de esperança, vergonha e impotência medeiam as ações de PS e nos estudantes com uma alta IP, somente a emoção de vergonha medeiam a participação. Finalmente discutem-se as implicações desses achados e as possibilidades e limitações que se apresentam para os estudantes universitários para participar no ME desde uma universidade privada.

Palavras-chave: Movimento estudantil; participação; emoções; identidade coletiva; identidade politizada; sociologia

$* * *$

\section{Introducción}

En Chile, los movimientos sociales (MS) han formado parte de toda su historia (Salazar, 2012). En especial, la lucha por la educación tiene data ya desde comienzos del siglo XX cuando miembros de las federaciones de estudiantes universitarios se involucran en los procesos de reforma del sistema universitario(Silva, Kronmüller, Cruz, y Riffo, 2016).Los ciclos más recientes de movilizaciones tienen sus antecedentes en el año 2006 con la denominada "revolución pingüina" conformada por estudiantes de educación secundaria, quienes organizados por la llamada Asamblea Coordinadora de Estudiantes Secundarios (ACES) solicitan, entre otras demandas, la gratuidad del pase escolar y de la Prueba de Selección Universitaria (PSU), y la derogación de la Ley Orgánica Constitucional de Enseñanza (L.O.C.E.). La L.O.C.E. creada en dictadura, plantea requisitos mínimos para la creación de instituciones educacionales sin velar por la calidad de la educación entregada y fomenta la búsqueda de los “mejores" estudiantes, es decir, instaura la competencia entre las instituciones educacionales para obtener mejores subsidios del Estado (Monje, 2019).

Más adelante, durante los años 2011 y 2012 se conforman nuevas movilizaciones estudiantiles masivas compuestas por estudiantes secundarios y universitarios, en busca de una "educación pública gratuita y de calidad”. Estas movilizaciones plantearon un cuestionamiento del modelo económico y social imperante en la educación en Chile, que durante las últimas décadas se ha caracterizado por un menoscabo de lo público a partir de la privatización de servicios como la salud o el sistema de pensiones, lo que bajo una lógica de mercado también convierte a la educación en un bien de consumo despojándola de su carácter de derecho social (Marambio, Gil de Montes y Valencia, 2015, Labbé, 2013).Este escenario planteado por el ME chileno conforma un ejemplo de un movimiento social (MS) en la cual interaccionan variables individuales y sociales que intentan explicar la participación de los estudiantes, lo cual ha sido objeto de estudio de dos disciplinas estrechamente vinculadas como la Sociología y Psicología Social (Ovejero, 2003). Aunque las demandas del ME fueron acogidas por el programa del segundo gobierno de Michel Bachelet (2014-2018) que buscaba fortalecer el sistema público y devolverle a la educación su estatus de derecho, el ME ha seguido activo persistiendo en la lucha por la calidad y equidad (Parker, 2017) sumándose distintos actores sociales, tales como docentes y estudiantes de universidades privadas que han mostrado distintos grados y formas de participación en estos procesos.

En el presente estudio adoptamos la perspectiva de la Psicología social, disciplina que presenta una tradición en la explicación del comportamiento colectivo y la participación social, y entiende al ser humano como un agente de cambio histórico que debe ser estudiado en contexto (Morales, 2007).El aporte de esta disciplina al estudio de las movilizaciones radica en que analiza la interacción entre procesos individuales y procesos sociales, considerando los aspectos cognitivos, afectivos, motivacionales y relacionales en estos contextos (Klandermans y Van Stekelenburg, 2007).De acuerdo con esta disciplina, se pretende analizar las relaciones entre las identidades colectivas y las emociones, con el fin de analizar su rol en el desarrollo y partici- 
pación de los MS. Aun cuando las teorías de corte más racional o cognitivo como la teoría de movilización de recursos (Johnston, 2003)esbozan que las emociones entorpecen el pensamiento racional, en las últimas décadas se ha resaltado su relevancia como motivadoras a la acción (Izard, 2007) y su aparición en contextos políticos (Alguacil, 2007; Bravo, 2010). Asimismo, se ha referido que constituyen un antecedente de los MS (Jaspers, 2006; 2012), relacionándose con la identidad de los individuos en contextos políticos (González, Manzi y Noor, 2013) y de movilización (Javaloy, 2003; Páez, Javaloy, Wlodarczyk, Espelt y Rimé, 2013).

\section{Movimientos sociales y participación social: una perspectiva psicosocial}

El surgimiento de un MS corresponde a un proceso o fenómeno situado resultado de los conflictos que ocurren en una determinada época histórica. Touraine (2006) señala que los factores más relevantes en el surgimiento de los movimientos sociales se basan en tres principios: identidad, entendido como el sentimiento de pertenencia al movimiento; oposición o la especificación del adversario contra el que lucha el movimiento; y totalidad, entendido como la definición de los objetivos a imponer.

Klandermans (2013) define la participación en los MS como un fenómeno multidimensional que se pueden expresar de diversas maneras, siendo fundamental para su análisis la consideración del tiempo y esfuerzo que las personas invierten en estas acciones. Para este autor, la participación puede obedecer a una razón instrumental, esto es, la participación por la búsqueda de cambio de circunstancias individuales, un espacio para expresar sentimientos, o una determinada posición respecto a un tema. Otra razón sería la identitaria donde el sujeto busca actuar como miembro de su grupo y en la cual la identificación con un grupo favorece la acción. Por ende, la mayoría de los estudios recientes sobre la acción colectiva tienen como objetivo integrar distintos enfoques teóricos tradicionales e incluir múltiples predictores de la participación política (e.g. Drury y Reicher, 2009; Gamson, 1992; Stürmer y Simon, 2004; Turner, 1987; Van Zomeren, Postmes, y Spears, 2008).

\section{Procesos identitarios vinculados a la participación}

Un MS o acción colectiva surge de la indignación moral que provoca una injusticia percibida por un endogrupo sobre el cual un "adversario" (endogrupo) actúa de manera ilegítima. Este importante hecho, es suficiente para generar una identificación compartida entre quienes vivencian la situación de injusticia (Paez et al., 2013).De acuerdo al Modelo Elaborado de la Identidad Social (ESIM) de Drury y Reicher, la identidad a conformar entre los miembros de un grupo y el contexto representan momentos interdependientes en un único proceso histórico, de tal modo que "la identidad constituye contexto y viceversa" (Drury y Reicher, 2009, p. 712). Asimismo, en un contexto de protesta, se generan dos representaciones antagónicas entre un grupo movilizado y otro externo (e.g., la policía) en la cual las acciones de uno u otro estarán determinadas por tales representaciones. Finalmente, el reposicionamiento que realiza el grupo externo sobre el grupo movilizado fomenta en éste último un nuevo sentido de identidad y formas de acción

En el presente estudio se adopta la perspectiva de la teoría de la identidad social (Tajfel y Turner, 1986) que se centra en el supuesto de que los individuos se perciben como parte del grupo más que como individuos independientes. De este modo, disminuyen su identidad personal y refuerzan la social. En este sentido, si el grupo se ve involucrado en un conflicto social se activa la identidad social, hasta tal punto que los miembros del movimiento se convierten en representantes de la sociedad y movimiento, más que personas únicas. Tajfel y Turner (1986) definen la IS como "aquella parte del autoconcepto del individuo que se deriva del conocimiento de su pertenencia a un grupo (o grupos) social junto con el significado emocional y valorativo asociado a dicha pertenencia” (p. 292).

La vinculación a un MS les brindaría a sus participantes un sentido de pertenencia e identidad grupal (Javaloy, 1993), aun cuando no haya un consenso entre sus integrantes acerca de ideologías, creencias, intereses u objetivos para reunirse. En este sentido, más que el acuerdo específico, se requiere que una percepción compartida respecto a la oposición frente a una situación dominante, lo cual proporcionaría la base para la consolidación de la identidad colectiva (Fominaya, 2010).

Según Melucci (1995) la Identidad Colectiva (IC) presenta las siguientes características: a) contiene 
definiciones cognitivas acerca de términos, significados y campos de acción a través de un lenguaje compartido, rituales y prácticas culturales; b) se compone de una red de relaciones activas entre actores en interacción, negociación y toma de decisiones, con unos canales y tecnologías de comunicación compartidos; y c) constituye una cierta inversión emocional que les da a los actores la capacidad de sentirse parte de una unidad común. Por otro lado, la identidad social politizada (IP) se refiere a una forma de IC que subyace a la disposición de los miembros del grupo a participar en una lucha de poder en términos sociales más amplios. Simon y Klandermans (2001) desarrollan el concepto de Identidad Colectiva Politizada (ICP)para identificar al proceso subyacente a la reacción en los miembros de un determinado grupo de participar de la acción cuando éstos son conscientes de una lucha de poder en medio de un contexto social más amplio en donde esta se suscita. Esta lucha es generalmente una transformación gradual de la relación del grupo con su entorno social. Según un meta-análisis de estudios sobre la acción colectiva realizado por Van Zomeren y Spears (2008) la IP fue el predictor más importante de la acción colectiva (registrándose tamaños de efecto más grandes que para las variables de injusticia, identidad y eficacia, las cuales predecían la acción colectiva de manera casi similar y con un tamaño de efecto moderado).

En cuanto a evidencias empíricas vinculadas a estos conceptos se ha encontrado una relación entre la ICP y el apoyo a protestas políticas en grupos étnicos (Wohl, King y Taylor, 2014). Otros autores han señalado que la politización es una fase más hacia la polarización y por consiguiente la radicalización del grupo, esto también resulta de interés para la acción colectiva debido a que, a mayor politización y polarización del grupo, mayor probabilidad de que se emprendan acciones colectivas radicales dirigidas a los opositores y la opinión pública para que tomen asuntos en sus demandas (Van Stekelenburg, 2014). Sin embargo, la evidencia no es definitiva, por ejemplo, se ha comprobado que la ICP puede predecir la acción política pacifista siempre que la angustia colectiva sea baja (Whol et al., 2014). Esto último, demuestra la importancia que tiene estudiar conjuntamente las emociones colectivas en al ámbito de la IC y la ICP.

\section{Emociones vinculadas a los MS}

El estudio psicosocial de las emociones las describe como respuestas desencadenadas frente a evaluaciones cognitivas del medio ambiente en el cual los individuos se desenvuelven (Morales, 2007), por lo que se consideran como construcciones sociales vinculadas a la sociedad y cultura donde se manifiestan (Belli y Iñiguez, 2008), y que pueden ser experimentadas de manera colectiva (Jaspers, 2006). Tras un largo periodo de haber ignorado o negado la importancia de las emociones, los actuales modelos explicativos de la acción colectiva incorporan emociones grupales (Stürmer ySimon, 2009; Thomas, McGarty, y Mavor, 2009; Van Stekelenburg, Klandermans, y Van Dijk, 2011; Van Zomeren, Spears, Fisher, y Leach, 2004; Van Zomeren, Leach, y Spears, 2012), particularmente la ira o indignación, como caminos distintos, pero complementarios a la acción colectiva (e.g. Mackie, Devos, y Smith, 2000; Smith y Mackie, 2008; Thomas, McGarty, y Mavor, 2009). Aminzade y McAdam (2002) argumentan que los procesos emocionales presentan una gran capacidad explicativa de los diversos MS. Si bien las emociones no representan el único factor que desencadena un MS, sin éstas no se generan. Jasper (2012) enfatiza que las emociones alientan a los individuos a la acción política y que cuando un individuo participa en un MS, está expuesto a nuevos procesos sociales que le ayudan a formar y moldear sus emociones. La propuesta de Jasper sobre la relevancia de la experiencia emocional en el contexto de los MS es compartida por variados autores (Johnston, 2003; Stürmer y Simon, 2009;Van Stekelenburg, Klandermans y Van Dijk, 2011), al identificarse que las emociones son socialmente funcionales y surgen de la experiencia de categorización social con un determinado grupo (Smith, Mackie 2019). Complementario a lo anterior, las evaluaciones y significados que los sujetos den a su situación respecto al contexto u otros grupos, determina el tipo o nivel de emoción que predomine en cada grupo (Sabucedo y Vilas, 2014).

En relación al tipo de emociones que impulsa a un MS, autores tales como Le Bon’s (1986) o Gamson (1992) plantearon que en los MS las emociones se limitaban a las negativas y principalmente a la ira, que proporcionaba la fuerza necesaria para las demandas colectivas. Sin embargo, tal movilización no está guiada únicamente por emociones negativas, sino que las emociones en general posibilitan la capacidad de evaluar la condición actual y futura de los individuos y, particularmente, los deseos de cambiarla. Además, se plantea la presencia de distintas emociones al considerar factores tales como los valores, liderazgos, narrativas, las propias instituciones, grupos con los que la movilización se desarrolla y las propias experiencias ocurridas en el 
proceso (Jarimowisc y Bar-tal, 2006).Coincidente con esto Becker, Taush y Wagner (2011) determinan que en el transcurso de unMS se generan emociones negativas y positivas en el individuo, asociadas a las relaciones con el endo y exogrupo. En relación al endogrupo, los individuos perciben más cohesión, sentido de pertenencia y esperanza, por el contrario, en relación al exogrupo experimentan emociones tales como ira o un sentimiento de injusticia.

Por ende, resulta sorprendente que tan pocos estudios han examinado directamente el papel de las emociones de valencia positiva. Aunque algunos estudios recientes señalan la importancia de considerar las emociones positivas en la explicación de las dinámicas grupales, gran parte de la literatura de acción colectiva no hace suficientemente hincapié en su potencial para motivar la participación en la protesta. Al respecto, Sabucedo y Vilas (2014) encuentran en grupos reivindicativos que si bien, generalmente se muestran airados por las injusticias del exogrupo, intra-grupalmente desarrollan condiciones que les permitan mantenerse deseosos de un futuro mejor para ellos.

Una de las emociones positivas que recientemente ha suscitado mayor interés es la esperanza (Braithwaite, 2004; Courville y Piper, 2004; McGeer, 2004). La esperanza es una emoción motivadora que es capaz de alimentar la resistencia y la protesta. Desde esta perspectiva la esperanza podría ser vista como un puente entre los marcos de acción colectiva y la acción real (Foster-Fishman, Cantillon, Pierce, y Van Egeren, 2007; Aminzade y McAdam, 2001, Frijda, 1988, Lazarus, 1991).El estudio de Wlodarczyk, Basabe, Páez y Zumeta (2017) exploró el rol de la esperanza y la ira como motores de la participación en acción colectiva, confirmando el papel crucial de la esperanza en movilizara los individuos. Además, Wlodarczyk et al. (2017) constataron que la influencia de las emociones negativas se realiza fundamentalmente a través de las emociones positivas (véase también Greenaway, Cichocka, van Veelen, Likki, y Branscombe, 2014; Páez, Javaloy, Wlodarczyk, Espelt, y Rimé, 2013; Sabucedo y Vilas, 2014; Smith y Leiserowitz, 2014).

Conjuntamente, se ha demostrado que tanto las emociones negativas, como las positivas juegan un rol importante en las dinámicas de protesta, y que es necesario distinguir entre las emociones que motivan a involucrarse en la acción colectiva y aquellas que están asociadas a un compromiso continuado (Cristancho, Gómez, y Wlodarczyk, 2014). Los resultados de los estudios anteriormente mencionados sugieren que la incorporación de las emociones al estudio de los MS, requiere un análisis de un espectro amplio de ellas tanto positivas como negativas para permitir un análisis exhaustivo del fenómeno de la acción política y abordar los cambios individuales dentro de un contexto cambiante.

\section{Sobre el presente estudio}

El presente estudio explora el ME chileno desde la perspectiva de la Psicología Social, relacionando acciones de participación vinculadas al ME con las emociones experimentadas ante la situación de la educación en Chile y la identidad de los estudiantes de una universidad privada. . En específico, la pregunta de investigación es: ¿Cómo una mayor presencia de emociones positivas y negativas se relaciona con la participación social de estudiantes universitarios chilenos de una universidad privada? A partir de lo antes expuesto, los objetivos específicos de este estudio son: primero, describir las frecuencias de las acciones de participación; segundo, explorar la relación entre las emociones, la IC e IP y las acciones de participación; tercero, analiza el rol mediador de las emociones en la relación entre la IC e IP y las acciones de participación. Se hipotetiza que quienes desplieguen acciones de participación lo harán sobre la base de emociones negativas como la ira o positivas como la esperanza. Como emociones negativas mediadoras se considerarían la ira, impotencia o la vergüenza (Páez et al., 2013) o emociones positivas como la esperanza (Páez et al., 2013; Sabucedo y Vilas, 2014).Además, las acciones de participación serán mayores en quienes se identifiquen más (IP)con las luchas de sus contextos sociales. Finalmente se espera que la relación entre sentirse identificado con las luchas sociales (IP) y el despliegue de acciones (PS) que estén en consonancia, este mediada tanto por emociones de valencia negativa como positiva.El planteamiento de tales variables independientes con la participación social resulta inédita, ya que habitualmente las variables explicativas se centran en la injusticia y eficacia (Páez et al., 2013; Valentino et al., 2009; Wlodarczyk et al., 2017) o incluso emociones propiamente tal (Sabucedo y Vilas, 2014). 


\section{Metodología}

\section{Muestra y procedimiento}

El muestreo fue por conveniencia, participando 224 estudiantes de pregrado de la carrera de Psicología de una universidad privada de la región del Biobío, correspondiendo a 94 hombres (41.96\%) y 126 mujeres (56,25\%) (rango de edad 19 a 33 años, $M=22.00 ; D S=2.11$ ). Con el fin de reclutar participantes para el presente estudio, en primer lugar, se contactó a los docentes pertenecientes a la universidad, con el fin de proponerles participación en el estudio y solicitar la posibilidad de aplicar el cuestionario en horario de clases. Un equipo de encuestadores previamente capacitados, invitó a los estudiantes a contestar el cuestionario, asimismo explicando que su participación era totalmente voluntaria. Los estudiantes que accedieron a participar firmaron el consentimiento informado y procedieron a contestar el cuestionario en formato papel.

\section{Instrumentos}

Acciones de participación en MS (basada en www.protestsurvey.eu; véase también Tausch, Becker, Spears, Christ, Saab, Singh, y Siddiqui 2011; y Van Zomeren et al., 2008). Se utilizó una versión de 6 ítems en formato tipo Likert, en el cual se solicitaba indicar con qué frecuencia se realizaron acciones de participación durante el último año (búsqueda de información en medios masivos de comunicación, el conversar con conocidos o familiares sobre el ME, la participación en foros o debates relacionados, compartir en redes sociales información al respecto, participación en manifestaciones, entre otras). El formato de respuesta frecuencias va de 1 (nunca) y 5 (más de 20 veces). La consistencia interna de la escala fue de 0.88 .

Escalas de Identidad Colectiva y Politizada (basada en Van Zomeren, Spears, Fisher, y Leach,2004; para mayor información acerca de las propiedades psicométricas de las escalas véase también Van Zomeren et al., 2008; Van Zomeren,Leach y Spears, 2010).Para medir la identificación grupal de los participantes se midió: en primer lugar, una dimensión de Identidad Colectiva o grupal vinculada a ser estudiante a través de tres ítems: "Ser estudiante refleja una parte importante de ti", "Te identificas con los otros estudiantes" y "Sientes que tienes mucho en común con los otros estudiantes”; y una segunda dimensión de Identidad Politizada, de identificación con los protestantes a través de 3 ítems: “Te identificas con los estudiantes que participan en el movimiento estudiantil", "Sientes que tienes un vínculo con los estudiantes que se manifiestan” y "Te ves a ti mismo como parte del movimiento estudiantil”. El formato de todos los ítems fue de tipo Likert con un formato de respuesta de 7 puntos donde 1 corresponde a Naday 7 a Mucho. La consistencia interna para la IC fue de 0.84 , y de 0.93 para IP.

Escala de reacción emocional extendida (basada en Echebarría y Páez, 1989). El instrumento fue utilizado en varios estudios en diferentes contextos culturales siempre demostrado una buena fiabilidad y validez (véase Greenaway, Cichocka, Van Veelen, Likki, y Branscombe, 2014; Smith y Leiserowitz, 2014). La escala está compuesta por 11 ítems mediante los cuales se evaluó el grado en que los participantes experimentaron varios estados emocionales (ira, indignación, preocupación, ansiedad, vergüenza, miedo, impotencia, esperanza, culpa, sorpresa, optimismo)al pensar en la situación actual de la educación en Chile. El tipo de respuesta sigue un formato de escala Likert en la cual se les pidió que califican de 1 (nada) al 7 (mucho) si sentían diferentes emociones. Un ejemplo de los ítems que se tomarán en cuenta son la. La consistencia interna de la escala fue 0.78 . Para el impacto emocional negativo esta fue $0.75 \mathrm{y}$ para el positivo, 0.83 .

\section{Análisis de los Datos}

En primer lugar, calculamos frecuencias, el promedio y desviación estándar de todas las variables analizadas y estimamos las diferencias con $t$ de Student. Con el fin de reflejar las relaciones entre las variables se calcularon correlaciones $\mathrm{r}$ de Pearson. Además, se analizaron las correlaciones entre las distintas variables del estudio, mediante el coeficiente de correlación de Pearson. Por último, con el objetivo de contrastar las hipótesis de mediación, es decir, para evaluar en qué medida las emociones desempeñan un rol mediador con respecto a la IC eIP y las acciones de participación se utilizó el macro PROCESS de SPSS(Hayes, 2017).Todos los análisis estadísticos fueron realizados con el programa SPSS 21.0v. Se estableció la significancia estadística en un valor p ?0.05. 


\section{Resultados}

\section{Frecuencias de acciones de participación en ME}

Entre las acciones relacionadas con participación en ME, las que fueron reportados con mayor frecuencia por los estudiantes de la universidad privada fueron: conversaciones sobreME con amigos y familia $(M=$ $3.11 D S=1.22$, con un 5.6\% que no lo ha hecho) y búsqueda de información sobre el ME en medios de comunicación tradicionales ( $M=2.56, D S=1.25$, con el $21.5 \%$ que no lo ha realizado), seguido por: compartir en facebook u otra red social alguna noticia del $\mathrm{ME}(M=1.87, D S=1.15$, con un $49.5 \%$ que no lo ha hecho), asistir a manifestaciones vinculadas con el $\operatorname{ME}(M=1,77, D S=1.05$, con un $53.7 \%$ que no lo ha realizado), participar en un debate, foro que trate temas relacionados con el ME $(M=1.72, D S=1.03$, con un $56.5 \%$ que no lo ha realizado) y participar en los paros convocados por el ME $(M=1.55, D S=.93$, con un $64 \%$ que no lo ha llevado a cabo). En lo que se refiere a asimetría y curtosis, las acciones de participación social descritas son indicativas de una asimetría con sesgo positivo (con valores entre 23 y 2.07) y de curtosis leptocúrtica (con valores entre 1.41 y 4.27$)$.

Respecto a diferencias por variables socio-demográficas, se encontraron diferencias significativas en las acciones de participación social en ME por sexo $t(208)=2,31, p=.022$, con $M_{\text {hombre }}=13.55$ y $M_{\text {mujer }}=11.85$, lo que confirma los hallazgos en otros estudios que informa de mayores acciones de participación social en hombres que mujeres. No se encuentran diferencias significativas en acciones de participación social en ME por edad.

\section{Relaciones entre tipo de emociones y variables en estudio}

En la Tabla 1, se observan las relaciones entre distintas expresiones emocionales con IC, IP y las acciones de participación social en el ME. A nivel general, se confirma que emociones de valencia positiva y negativa impulsan las acciones de participación social en ME, relación que también se establece con la IP. Aunque la relación es significativa en todos los tipos de expresión emocional, en ambos casos, es mayor con las emociones del tipo negativas-activas y emociones morales. Asimismo, es llamativa que la variable de IC presenta una alta significación fundamentalmente con las emociones positivas y las emociones negativas-activas.

Tabla 1

Correlaciones entre distintos tipos de emociones y variables en estudio

\begin{tabular}{lccc}
\hline \multicolumn{1}{c}{ Tipos de emociones } & IC & IP & PS \\
\hline Ira $^{1}$ & $.193^{* *}$ & $.445^{* *}$ & $.437 * *$ \\
Indignación $^{1}$ & $.144^{*}$ & $.409 * *$ & $.431^{* *}$ \\
Impotencia $^{2}$ & $.192^{* *}$ & $.409 * *$ & $.345^{* *}$ \\
Sorpresa $^{2}$ & $.187^{* *}$ & $.287 * *$ & $.328^{* *}$ \\
Miedo $^{2}$ & .067 & $.338^{* *}$ & $.299 * *$ \\
Preocupación $^{2}$ & $.259^{* *}$ & $.453^{* *}$ & $.426 * *$ \\
Verguenza $^{3}$ & $.157^{*}$ & $.338^{* *}$ & $.379 *$ \\
Culpa $^{3}$ & .022 & $.194 * *$ & $.200^{* *}$ \\
Esperanza $^{4}$ & $.227^{* *}$ & $.305^{* *}$ & $.238^{* *}$ \\
Optimismo $^{4}$ & $.207^{* *}$ & $.241^{* *}$ & $.176^{*}$ \\
\hline
\end{tabular}

Nota: ${ }^{*} p<.05, * * p<.01$

IC= identidad colectiva; IP= identidad política; PS= participación social

1. Emociones negativas activas; 2 Emociones negativas pasivas; 3 Emociones morales;

4. Emociones positivas 
Una correlación adicional entre las acciones de participación social en ME y los dos tipos de identidades descritos en este estudio, revela diferencias significativas, pero con variación, puesto que las acciones de participación social en ME se asocia positivamente con IP $(r=.65, p<.001)$ e IC $(r=.27, p<.001)$. Esto plantea que los universitarios perciben una IC como estudiantes que demandan cambios, pero aquellos que se sienten más parte del ME, se sienten removidos desde una postura política.

\section{Análisis de mediación}

Se realizaron dos tipos de análisis de mediación: primero, para identificar qué emociones eran un factor mediador entre la IC y acciones de participación social (ver Figura 1). Se realizaron dos tipos de análisis de mediación: primero, para identificar de qué modo las emociones negativas como la indignación, impotencia o vergüenza o las emociones positivas como la esperanza eran un factor mediador entre la IC y acciones de participación social. Los resultados se presentan en la Figura1, que identifican tanto a la esperanza, vergüenza e impotencia como variables intervinientes en la relación entre la IC y las acciones de participación social, descartándose la indignación. Se encontró que, en primer lugar, la IC se asoció positivamente con las acciones de participación social $(\mathrm{B}=.37, t(211)=4.12, p=.0001)$. Asimismo, se halló que la IC se relacionó positivamente con la emoción de esperanza $(\mathrm{B}=.11, t(211)=3.67, p=.0003)$, con la emoción de vergüenza $(\mathrm{B}=.08$, $t(211)=2.31, p=.02)$ y con la emoción de impotencia $(\mathrm{B}=.10, t(211)=3.10, p=.002)$. Conjuntamente, la emoción de esperanza se relacionó positivamente con las acciones de participación social $(\mathrm{B}=.46, t(211)=$ $2.46, p=.01)$, así como la vergüenza con acciones de participación social $(\mathrm{B}=.64, t(211)=3.53, p=.0005) \mathrm{y}$ la impotencia con acciones de participación social $(\mathrm{B}=.44, t(211)=2.40, p=.01)$. A partir de la mediación de las variables intervinientes, el efecto directo de la IC con las acciones de participación social siguió siendo significativa $(\mathrm{B}=.21, t(211)=2.50, p=.01)$, aunque conforma un modelo de regresión parcial. Las variables incluidas en este modelo explican el $22 \%$ de la varianza

Figura 1

Modelo de mediación parcial entre la identidad colectiva y las acciones de participación social en ME, a través de las emociones de esperanza, vergüenza e impotencia

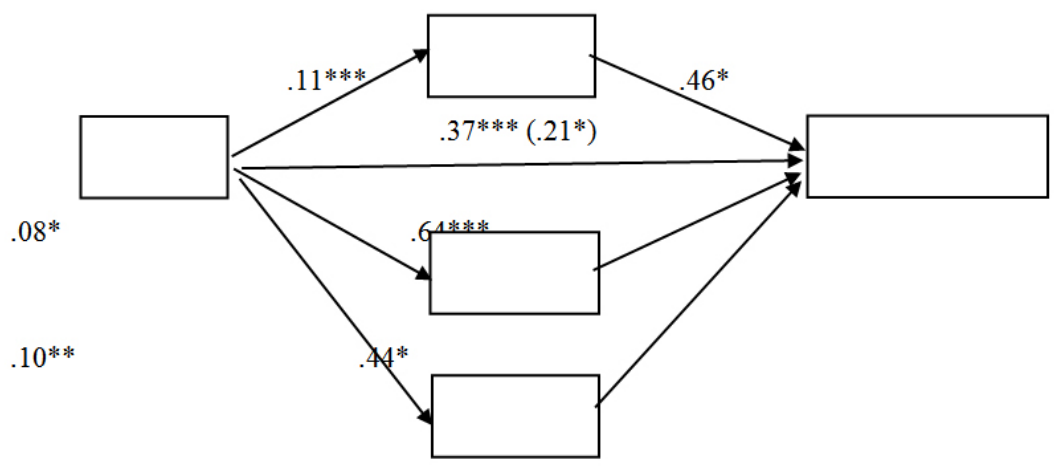

Nota: Se presentan los coeficientes de regresión B no estandarizados. IC= identidad colectiva; PS= participación social ${ }^{*} p<.05,{ }^{* *} p<.01,{ }^{* * *} p<.001$

En segundo lugar, se realizó igualmente un modelo de mediación para identificar qué emociones intervienen entre la IP y las acciones de participación social en ME. En la Figura 2 se muestra que la IP presenta una relación significativa con las acciones de participación social $(\mathrm{B}=.64, t(210)=12.68, p=.000$. Luego, la IP se relacionó significativamente con la emoción de vergüenza $(\mathrm{B}=.12, t(210)=5.25, p=.000)$. Esta última variable se establece como mediadora de las acciones de participación social $(\mathrm{B}=.474, t(210)=3.18, p=.001)$. Conjuntamente, los resultados indicaron que el efecto directo de la IP con las acciones de participación social 
siguió siendo significativa $(\mathrm{B}=.58, t(210)=11.08, p=.000)$, lo que sugiere una mediación parcial. Estas variables explican el $45 \%$ de la varianza del modelo de las acciones de participación social. Estos resultados identifican a la vergüenza como la emoción que media entre ambos tipos de identidad y las acciones de participación social.

Figura 2

Modelo de mediación parcial entre la identidad politizada y las acciones de participación social en ME, a través de la Vergüenza

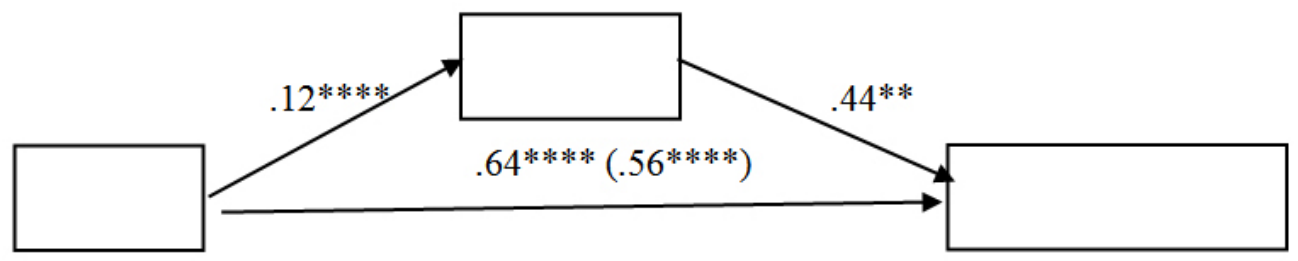

Nota: Se presentan los coeficientes de regresión B no estandarizados. $\mathrm{IP}=$ identidad politizada; $\mathrm{PS}=$ participación social $* * p<.01, * * * * p<.0001$

\section{Discusión y conclusiones}

El estudio describe las acciones de participación política en el ME de estudiantes de una universidad privada, temática que resulta inédita en el actual panorama de las investigaciones de movimientos sociales y del ME en particular. Los hallazgos confirman que la participación de hombres es mayor que en las mujeres, tal como lo han planteado otros estudios en otros contextos de movilización social (Páez et al., 2013), lo que revela que posiblemente los hombres presentan condiciones que facilitan su participación o como explican Cicognani, Pirini, Keyes, Joshanloo, Rostami, y Nosratabadi (2008) estas diferencias podrían reflejar aún la existencia de expectativas tradicionales de los roles de género, donde los hombres tradicionalmente se han visto más "activos” con respecto a la participación social o sus acciones en el contexto de los MS han tenido mayor visibilidad. En específico en relación al contexto universitario privado, los estudiantes se orientan a acciones de participación política que implican menor esfuerzo (i.e., tales como conversar con familia y amigos del ME o la búsqueda de información del movimiento en medios de comunicación tradicionales), en comparación a otras acciones que requieren mayor esfuerzo (i.e., postear información en redes sociales o participar en foros) o asumir una posición política afín al ME (i.e., participar u organizar un paro).

En este estudio, tales acciones de participación política son contrastadas a partir de la percepción de IC, identidad política y emociones de los estudiantes universitarios. Los procesos de identidad fomentan mayor identificación con los MS y aquí se confirma que la existencia de una IC de sentirse estudiante universitario en Chile, independientemente del tipo de institución universitaria, junto con una identidad política de adherirse mediante acciones políticas a un ME. En este punto, en relación con las acciones de participación política, resultaría de mayor influencia cuando los estudiantes de una universidad presenten una ideología o se sienten afines a los objetivos políticos que plantea el movimiento. Al respecto, los resultados muestran que los últimos estudiantes que manifiesta una alta identificación con el ME conforman un grupo menos frecuente en la universidad privada, pero a la vez son quienes realizan acciones de participación política de mayor esfuerzo o que se orientan a un fin político.

Sobre la base de las teorías de la Psicología Social en relación al papel de las emociones en la participación social, predijimos que la ira o la esperanza intervienen en la relación entre la IC y las acciones de participación social, en una relación de variables independiente y dependiente que no ha sido explorada anteriormente en otras investigaciones. Esta predicción se confirma parcialmente, en el sentido que las emociones de vergüenza y la esperanza tienen una alta incidencia en acciones de participación social (Páez et al., 2013; Wlodarczyk 
et al., 2017). No obstante, no se confirma la emoción de la ira que se identifica igualmente en ambos estudios y en un estudio longitudinal en estudiantes (Tausch y Becker, 2013). En este sentido, se plantea que la impotencia que sí se observa como variable moderadora en el presente estudio, responde a una emoción que posiblemente se encuentre más asociada a una emoción intergrupal de mayor durabilidad que la rabia (véase RodriguezHernández, Juárez-Lugo y Ponce de León, 2012). Esto pone de manifiesto que tales emociones, aparentemente contradictorias, dependen del contexto universitario que presentan los estudiantes para participar. Al contrastar estos resultados con los hallazgos de Triani (2009) que muestran que la vergüenza en combinación con la ira o la indignación moral serían un antecedente para un compromiso más activo con los MS, se podría entender que las emociones que manifiestan los estudiantes de la universidad privada se relacionen con acciones de participación de "menor" implicación. La aparición de la esperanza como mediador también deja la puerta abierta para hipotetizar que quizás esta combinación de valencia de emociones pueda ser antecedente de la implicación de los estudiantes de universidades privadas en las movilizaciones más recientes.

Por otro lado, en relación a la IP y las acciones de participación social planteada, únicamente la vergüenza aparece como variable mediadora. Esto requiere una posible explicación: de acuerdo a Hansberg (1996) sentir vergüenza, implica creencias sobre el propio status y de un observador. Primero, de sí mismo, un sujeto se siente en desventaja de lo que debería ser y, segundo, en este sujeto subyace la idea de que es visto de manera inadecuada por un observador. La vergüenza, además, constituye una emoción moral que surge cuando están en juego los valores, ideales y exigencias morales de cómo ser y vivir para tal sujeto (Hansberg, 1996). Esto, extendiéndolo a un contexto de ME, plantearía que un estudiante presenta una noción de desventaja que lo pone en similares condiciones con otros (IP) aspecto que es elicitado mediante la vergüenza. Asimismo, mediante la vergüenza, los estudiantes se orientan a proteger su dignidad, lo que posibilitaria, por tanto, las acciones de participación social por un cambio.

Otra fundamentación que podría abordar los resultados encontrados aquí es a través del mencionado modelo ESIM (Drury y Reicher, 2009) y se podría sintetizar del siguiente modo: el sentido de la posición social (identidad social) de los estudiantes se va modificando y acrecentando en la medida en que, al actuar sobre su identidad en acciones del ME, son reposicionados como efecto de las consideraciones y reacciones de autoridades o actores institucionales. Tales actores tenderían a criticar las acciones de los estudiantes lo que retroalimentaría a que éstos últimos se orienten a un nuevo sentido de identidad y a nuevas formas de participación social.

En este punto, cabe referir respecto al acceso y al perfil de los estudiantes de la universidad privada estudiada: el ingreso se ha diversificado en estos últimos años, lo que posibilita una mayor incorporación de estudiantes, puesto que disponen de sistemas de becas y ayudas económicas que la propia universidad establece o a través de los beneficios que proporciona el Estado chileno (e.g., becas del Ministerio de Educación o Crédito con Garantía del Estado). Respecto a las características de los estudiantes, provienen de estratos socioeconómicos bajos, que provienen de un sistema de educación secundaria en Chile en el cual persiste la segmentación social y educativa (Baeza, 2006). Esto fomenta que los estudiantes secundarios se proyecten casi exclusivamente a través de la educación universitaria y, para quienes provienen de una educación previa municipal, les condicione la elección de sus estudios (Puga, Atria, Fernández y Araneda, 2017). Esto, sumado a una escasa o nula experiencia previa en organización o participación social en el periodo secundario (DiarioUChile, 2018), podría explicar que haya una menor orientación a identificar necesidades como colectivo para abordar los problemas que puedan aquejar a estudiantes universitarios en un contexto privado.

Asimismo, la educación universitaria privada en Chile ha sido fuertemente asociada a un modelo neoliberal que cuestiona su calidad (para una mayor discusión, véase Espinoza, 2017). Un aspecto a analizar aquí se refiere a las condiciones que definen las propias universidades privadas en la participación social de los estudiantes. Un primer planteamiento podría asociar que la organización de los estudiantes en universidades privadas no está del todo estructurada como la de universidades públicas, que presenta décadas de experiencia en federaciones y que, paulatinamente, los estudiantes de universidades privadas están generando cambios. En este sentido, se observa la necesidad de que los estudiantes cuenten con la información necesaria para generar los espacios de organización, que fomente una acción política y participativa en estos contextos. Por el contrario, se ha señalado que los estudiantes universitarios reciben presiones cuando deciden movilizarse (DiarioUChile, 
2018) fomentando que una mayoría de éstos no participe. Incluso, se ha producido que algunos planteles de estudiantes hayan sido afectados con sumarios en los cuales se castiga tal participación, lo que redunda en que los contextos universitarios privados se plantean acciones contrarias a la participación y organización estudiantil. Este contexto descrito resulta clave para entender los hallazgos del presente estudio y plantea diversos desafíos respecto a cómo comprender y fomentar una cultura de participación social de los estudiantes en universidades privadas.

De allí que la diversidad de emociones que se relacionan con las acciones de participación social de los estudiantes universitarios responderán a cómo están percibiendo el contexto y las respuestas de las autoridades de la universidad privada: en algunos, la emoción de la esperanza representa una oportunidad de participar en movilizaciones que le resultan inédita. De otro lado, la impotencia constituye una emoción negativa pasiva asociado a un estado de indefensión; en estudiantes que perciben tal indefensión en el contexto universitario les promueve a identificarse colectivamente con los estudiantes y los impulsa a realizar acciones afines al ME. En tanto la vergüenza, como emoción moral, representa aquel estado de sensibilidad ante los problemas sociales y el impulso activo a desarrollar acciones sociales y, fundamentalmente, políticas. Esto último sugiere que los estudiantes que sienten más vergüenza son los más orientados a desarrollar las acciones de movilización, independientemente de las limitaciones que plantea el contexto universitario privado.

\section{Limitaciones y futuros estudios}

Dado que el presente estudio analiza las acciones de participación política en ME en estudiantes de una universidad privada, hubiese sido de interés comparar con una muestra de estudiantes de universidad pública, de tal modo de evidenciar las posibles diferencias que facilitarían u obstaculizarían tales acciones en un tipo y otro de universidad. Asimismo, el estudio se centra únicamente en estudiantes de la carrera de Psicología, no incorporando otras carreras que posiblemente presenten repertorios diversos de acciones de participación política. Tal como se ha señalado con anterioridad, las posibilidades de iniciar y mantener acciones de participación política tiene relación a los contextos institucionales que fomenten o restrinjan tales acciones. Por otro lado, el estudio identifica acciones de participación en un momento particular, las cuales requieren ser exploradas en el tiempo, de tal modo que se reconozcan aquellas acciones que se mantienen a lo largo de la vida universitaria o aquellas que son más transitorias.

Futuros estudios requieren continuar explorando cómo ocurre la participación política de estudiantes en universidades privadas, en específico, describir qué contenidos están formando parte de sus demandas o, por ejemplo, resaltar las acciones de organización y/o resistencia de los estudiantes frente a las condiciones institucionales. Esto plantea un campo de investigación menos conocido que es necesario frente a la necesidad de explorarlos distintos escenarios que plantea las reformas educativas en el contexto universitario en Chile. Complementariamente, dado que también han ocupado una posición en las movilizaciones, resulta de interés explorar las percepciones de los docentes, poniendo en evidencia justamente la dinámica establecida con los estudiantes que están participando de la ME y qué elementos sobresalen en tal dinámica. Por último, cabría explorar si los jóvenes estudiantes adquieren un empoderamiento que perdure más allá de estas acciones colectivas en este periodo (Drury y Reacher, 2005), de tal forma si motiva una participación en futuras acciones colectivas.

\section{Bibliografía}

Agacino, R. (2012). Movilizaciones sociales: coyuntura y aperturas políticas del período. Plataforma Nexos.

Aminzade, R., y McAdam, D. (2002).Emotions and contentious politics. Mobilization: an international quarterly, 7(2), 107-109.

Alguacil Gómez, J. (2007) Nuevos movimientos sociales: nuevas perspectivas, nuevas experiencias, nuevos desafios, Polis, (17). doi: 10.32735/S0718-6568/2007-N17-518 
Baeza, J. (2006). Demandas y organización de los estudiantes secundarios: una lectura sociológica más allá de fronteras y análisis coyunturales. Revista Temas Sociológicos, (11), 263-298.

Becker, J., Tausch, N., y Wagner, U. (2011). Emotional consequences of collective action participation differentiating self-directed and outgroup-directed emotions. Personality and Social Psychology Bulletin, 37(12), 15871598

Braithwaite, V. (2004). The hope process and social inclusion. The Annals of the American Academy of Political and Social Science, 592, 128.doi: 10.1177/0002716203262096

Bravo, N. (2010). Movimientos Sociales y acción colectiva como bases de la filosofía latinoamericana. Polis, 27.doi:

Castells, M. (2012). Networks of Outrage and Hope: Social Movements in the Internet Age. Cambridge, UK: Polity Press.

Cicognani, E., Pirini, C., Keyes, C., Joshanloo, M., Rostami, R., y Nosratabadi, M. (2008). Social participation, sense of community and social well being: A study on American, Italian and Iranian university students. Social Indicators Research, 89(1), 97-112.

Courville, S., y Piper, N. (2004).Harnessing hope through NGO activism. The Annals of the American Academy of Political and Social Science, 592, 39.doi: 10.1177/0002716203261940

Cristancho, C., Gómez, C., y Wlodarczyk, A. (2014) Dos años de indignación: cambios en las motivaciones y actitudes en el 15M.Una mirada transdisciplinar del 15M, 1, 490-511.

Drury, J., y Reicher, S. (2005). Explaining enduring empowerment: A comparative study of collective action and psychological out comes. European Journal ofSocialPsychology, 35(1), 35-58.

Drury, J., y Reicher, S.D. (2009). Collective psychological empowerment as a modelofsocialchange: Researchingcrowdsand power. Journal of SocialIssues, 65(4), 707-726. doi: 10.1111/j.15404560.2009.01622.x

diarioUchile. (2017). Estudiantes de universidades privadas: La voz de los invisibles. Edición 5 de junio de 2018. Disponible en: https://radio.uchile.cl/2018/06/05/estudiantes-de-universidades-privadas-la-voz-de-los-invisibles/

Echebarría, A. y Páez, D. (1989). Emociones: perspectivas psicosociales. Madrid, España: Fundamentos.

Espinoza, Ó. (2017). Privatización de la educación superior en Chile: consecuencias y lecciones aprendidas. EccoS Revista Científica, (44), 175-202.

Greenaway, K. H., Cichocka, A., van Veelen, R., Likki, T., y Branscombe, N. R. (2016). Feeling hopeful inspires support for social change. Political Psychology, 37(1), 89-107.

Fominaya, C. F. (2010). Creating cohesion from diversity: The challenge of collective identity formation in the global justice movement. Sociological Inquiry, 80(3), 377-404.

Foster-Fishman, P. G., Cantillon, D., Pierce, S. J., y Van Egeren, L. (2007). Building an active citizenry: The role of neighborhood problems, readiness, and capacity for change. American Journal of Community Psychology, (39), 91-106.doi: 10.1007/s10464-007-9097-0

Frijda, N. H. (1988). The laws of emotion.American Psychologist, 43(5), 349-358. 
Gamson, W. A. (1992). Talking politics. Cambridge, UK: Cambridge university press.

Gaudichard, F. (2011). Reflexiones sobre el “largo mayo chileno”. Cuando el neoliberalismo triunfante se agrieta Herramienta WEB 10. Recuperado de http://www.herramienta.com.ar/herramienta-web- 10/reflexiones-entorno-al-largo-mayo-chileno- cuando-el-neoliberalismo-triunfante-se

González, R., Manzi, J., y Noor, M. (2013). Identidad social y emociones intergrupales: antecedentes de las actitudes de perdón y reparación política en Chile. Psykhe, 22(2), 129-146.

Greenaway, K. H., Cichocka, A., van Veelen, R., Likki, T., yBranscombe, N. R. (2014).Feeling Hopeful Inspires Support for Social Change. Political Psychology. doi: 10.1111/pops.12225

Hanna, F. J. (2002).Therapy with difficult clients: Using the precursor's model to awaken change. Washington DC, USA: American Psychological Association.

Hasnberg, O. E. (1996). De las emociones morales. Revista de Filosofía, $3^{\circ}$ época, IX(16), 151-170.

Hayes, A. F. (2017). Introduction to mediation, moderation, and conditional process analysis: A regression-based approach. New York: Guilford Publications.

Hunt, S. A., yBenford, R. D. (2004). Collective identity, solidarity, and commitment. The Blackwell companion to social movements, 433, 57.

Izard, C. E. (2007). Basic emotions, natural kinds, emotion schemas, and a new paradigm. Perspectives on psychological science, 2(3), 260-280.

Jarymowicz, M., y Bar?Tal, D. (2006). The dominance of fear over hope in the life of individuals and collectives. European Journal of Social Psychology, 36(3), 367-3

Jasper, J. M. (2012). ¿De la estructura a la acción? La teoría de los movimientos sociales después de los grandes paradigmas. Sociológica (México), 27(75), 7-48.

Jasper, J. M. (2006).Motivation and emotion.En Goodin, R. y Tilly, C. (Ed.), The Oxford handbook of contextual political analysis.doi: 10.1093/oxfordhb/9780199270439.003.0008

Johnston, H. (2003). El reencuentro de la Psicología Social con la conducta colectiva y los movimientos sociales. Revista de Psicología Social, 18(2), 207-211

Javaloy, F. (1993). El paradigma de la identidad social en el estudio del comportamiento colectivo y de los movimientos sociales. Psicothema (5), 277-286.

Javaloy, F., Rodrì̀guez, A. y Espelt, E. (2001). Comportamiento colectivo y movimientos sociales. Madrid, España: Prentice Hall.

Klandermans, P. (2013). Identity Politics and Politicized Identities: Identity Processes and the Dynamics of Protest. Political Psychology, 35(1), 1-22.doi:10.1111/pops.12167

Klandermans, B. (1997). The social psychology of protest. Cambridge, UK: Blackwell.

Klandermans, B., y Van Stekelenburg, J. (2007).Individuals in movements.A social psychology of contention. En B. Klandermans y C. Roggeband (Eds.), Handbook of social movements across disciplines.New York, USA: Springer science.

Labbé, J. F. (2013). Movimiento estudiantil en Chile (2011): Repertorios de acción, marcos de acción colectiva, 
impactos y desafíos para la política. Revista de Ciencias Sociales del IUIOG, 11(31), 1-9. Recuperado de http://www.ortegaygasset.edu/publicaciones/circunstancia/ano-xi-n-31-mayo-2013/articulos/movimiento-estudiantil-en-chile-2011—repertorios-de-accion—marcos-de-accion-colectiva—impactos-y-desafiospara-la-politica-publica-

Lazarus, R. S. (1991). Emotion and adaptation. New York, USA: Oxford University Press.

Lerner, J.S., y Keltner, D. (2001). Fear, anger, and risk.Journal of Personality and Social Psychology, (81), 146159

Mackie, D.M., Devos, T., y Smith, E.R. (2000). Intergroup emotions: Explaining offensive action tendencies in an intergroup context. Journal of Personality and Social Psychology, (79),602-616.

Marambio, K., Gil, L. y Valencia, J. (2015). Representaciones sociales, inteligencia y conflicto de la educación en Chile. Psykhe, 24(1), 1-11.

Morales, F. (2007).Psicología Social. Madrid, España: McGrawHill.

McGeer, V. (2004).The art of good hope.The ANNALS of the American Academy of Political and Social Science, 592, 100.doi: 10.1177/0002716203261781

Monje, J. (2019). Políticas públicas de educación infantil en Chile y Brasil: tensiones y tendencias sobre calidad y gestión local. Paulo Freire, (20), 181-201.

Melucci, A. (1995). The process of collective identity. Social movements and culture, (4), 41-63.

Ovejero, A. (2003). Psicología colectiva y compromiso. Revista de Psicología Social, 18(2), 213-221.

Parker, C. (2017). Chile: la sociedad civil en movimiento frente al modelo neoliberal. CETRI, Centre Tricontinental.Recuperado de: https://www.cetri.be/Chile-la-sociedad-civil-en?lang=fr

Páez, D., Javaloy, F., Wlodarczyk, A., Espelt, E., y Rimé, B. (2013). El movimiento 15-M: sus acciones como rituales, compartir social, creencias, valores y emociones. Revista de Psicología Social, (28), 19-33.

Peìrez Navarro, C. (2018). Reconstruccioìn del proceso de elaboracioìn de la Ley Orgaìnica Constitucional de Ensenbanza: actores, proyectos y disputas ideoloìgicas. Chile, 1973-1990. Espacio, Tiempo y Educacioin, 5(2), pp. 179-195. DOI: http://dx.doi.org/10.14516/ete.170

Puga, I., Atria, R., Fernández, R., y Araneda, C. (2017). Proyectos de vida y oportunidades en la educación media. Nuevas demandas sociales al sistema escolar chileno. Última Década, (47), 118-153.

Rodríguez-Hernández, G., Juárez-Lugo, C. y Ponce de León, M.C. (2012). La culturalización de los afectos: Emociones y sentimientos que dan significado a los actos de protesta colectiva. Revista Interamericana de Psicología/Interamerican Journal of Psychology, 45(2), 193-202.

Salazar, G. (2012). Movimientos sociales en Chile. Trayectoria histórica y proyección política. Santiago, Chile: Uqbar.

Sabucedo, J., Durán, M. y Alzate, M. (2010). Identidad colectiva movilizada. Revista de Psicología Social, 25(2), 189- 201.

Sabucedo, J. y Vilas, X. (2014). Anger and positive emotions in political protest.UniversitasPsychologica, 13(3), 829-838. 
Silva, C., Kronmüller, C., Cruz, M., y Riffo, I. (2016). Empoderamiento en el Movimiento Estudiantil, Años 20112012 en Chile. UniversitasPsychologica,14(4), 1299-1310.

Simon, B., yKlandermans, B. (2001). Politicized collective identity: A social psychological analysis. American psychologist, 56(4), 319.

Smith, N., yLeiserowitz, A. (2014).The Roleof Emotion in Global WarmingPolicy Support and Opposition. Risk Analysis, (34), 937-948. doi: 10.1111/risa.12140

Smith, E. R., yMackie, D. M. (2008). Intergroup emotions. In M. Lewis, J. Haviland-Jones, y L. F. Barrett (Eds.), Handbook of Emotions (pp. 428-439). New York, USA: Guilford.

Stürmer, S., y Simon, B. (2004). The role of collective identification in social movement participations: A panel study in the context of the German gay movement. Personality y Social Psychology Bulletin, (30), 263-277.

Stürmer, S. y Simon, B. (2009). Pathways to Collective Protest: Calculation, Identification, or Emotion? A critical analysis of the role of group-based anger in social movement participation.Journal of Social Issues,(69), 681-705

Tajfel, H., y Turner, J. (1986). JC (1986).The social identity theory of intergroup behavior. Psychology of intergroup relations, $7-24$.

Tausch, N., Becker, J., Spears, R., Christ, O., Saab, R., Singh, P., y Siddiqui, R. N. (2011). Explaining radical group behaviour: Developing emotion and efficacy routes to normative and non-normative collective action. Journal of Personality and Social Psychology,101, 1, 129-148.

Tausch, N., y Becker, J. C. (2013). Emotional reactions to success and failure of collective action as predictors of future action intentions: A longitudinal investigation in the context of student protests in Germany. British Journal of Social Psychology, 52(3), 525-542

Thomas, E. F., McGarty, C., yMavor, I. (2009a). Aligning identities, emotions, and beliefs to create commitment to sustainable social and political action. Personality and Social Psychology Review, (13),194-218.

Thomas, E. F., McGarty, C., yMavor, K. I. (2009b). Groups in action: Social identity and norms about emotion and efficacy in the fight against poverty in developing countries.

Touraine, A. (2006). Los movimientos sociales. Revista colombiana de Sociología, (27), 255-278

Traini C. (2009). Emotions...mobilizations.Pariìs, France: Presses de Science Po.

Turner, R. y Killian, L. (1987). Collective behavior. Englewood Cliffs, USA: Prentice Hall.

Urzúa, S. (2015). ¿Cómo marchan los jóvenes en el Chile de postdictadura?: Algunas notas acerca de la apropiación del espacio público y el uso político del cuerpo. Última década, (23), 39-64.

Valderrama, L. B. (2013). Jovenes, Ciudania Y Tecnologia de Informacion y Comunicación. El movimiento estudiantil chileno. Revista Latinoamericana de Ciencias Sociales, Niñez y Juventud, 11(1), 123-135. Recuperado de http://eds.b.ebscohost.com/ehost/detail/detail?vid=30ysid=d52bdfc7-2136-4cd2-bcf36af11e8be19c\%40sessionmgr112yhid=120ybdata=Jmxhbmc9ZXMmc210ZT1laG9zdC1saXZl\#AN=86250428ydb=fua

Van Stekelenburg, J. (2014). Going all the way: Politicizing, polarizing, and radicalizing identity offline and online. Sociology Compass, 8(5), 540-555.

Van Stekelenburg, J., Klandermans, B., y van Dijk, W.W (2011). Combining motivations and emotion: The 
motivational dynamics of protest participation. Revista de Psicología Social: International Journal of Social Psychology, 26(1), 91-104. doi:10.1174/021347411794078426

Van Stekelenburg, J., yKlandermans, B. (2013). The social psychology of protest.Current Sociology, 61(5-6), 886905.doi:10.1177/0011392113479314

Van Zomeren, M., Leach, C. W., y Spears, R. (2010). Does group efficacy increase group identification? Resolving their paradoxical relationship. Journal of Experimental Social Psychology, 46, 1055-1060.doi: 10.1016/ j.jesp.2010.05.006

Van Zomeren, M., Leach, C. W., y Spears, R. (2012). Protesters as „Passionate Economists“: A dynamic dual pathway model of approach coping with collective disadvantage. Personality and Social Psychology Review, (16), 180-198.doi: 10.1177/1088868311430835

Van Zomeren, M., Postmes, T., y Spears, R. (2008). Toward an integrative Social Identity Model of Collective Action: A quantitative research synthesis of three socio-psychological perspectives. Psychological Bulletin, 134, 504-535.doi: 10.1037/0033-2909.134.4.504

Van Zomeren, M., Saguy, T. y Schellhaas, F. (2012). Believing in "making a difference" to collective efforts: Participative efficacy beliefs as a unique predictor of collective action. Group Processes YIntergroup Relations, 16, 618-634.doi: 10.1177/1368430212467476

Van Zomeren, M., Spears, R., Fischer, A. H., y Leach, C. (2004). Put Your Money Where Your Mouth Is! Explaining Collective Action Tendencies Through Group-Based Anger and Group Efficacy. Journal of Personality and Social Psychology, 87(5), 649-664.doi: 10.1037/0022-3514.87.5.649

Wlodarczyk, A., Basabe, N., Páez, D., yZumeta, L. (2017).Hope and anger as mediators between collective action frameworks and participation in collective mobilization: the case of 15-M. ?Journal of Social and Political Psychology, 5(1), 200-223,

Wohl, M., King, M., y Taylor, D. (2014).Expressions of political practice: Collective angst moderates politicized collective identity to predict support for political protest (peaceful or violent) among diaspora group members. International Journal Of Intercultural Relations, 43, 114-125.doi:10.1016/j.ijintrel.2014.08.020 